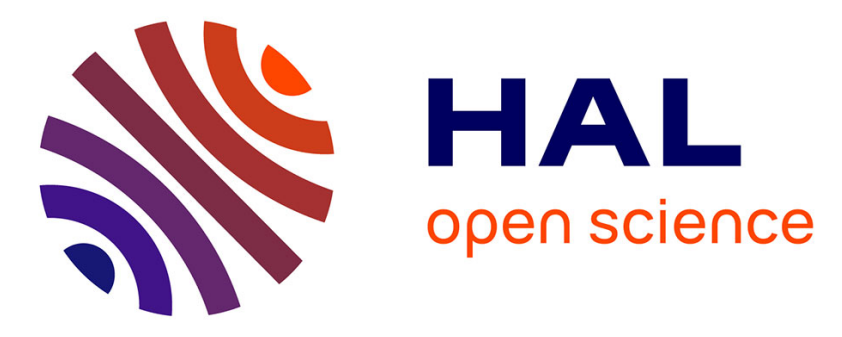

\title{
Delay-induced instability in phase-locked dual-polarization distributed-feedback fiber lasers
}

Marie Guionie, Marco Romanelli, Aurélien Thorette, Anthony Carré, Emmanuel Pinsard, Laurent Lablonde, Benoît Cadier, Mehdi Alouini, Marc Vallet, Marc Brunel

\section{To cite this version:}

Marie Guionie, Marco Romanelli, Aurélien Thorette, Anthony Carré, Emmanuel Pinsard, et al.. Delayinduced instability in phase-locked dual-polarization distributed-feedback fiber lasers. Physical Review A, 2020, 101 (4), pp.043843. 10.1103/PhysRevA.101.043843 . hal-02565340

\section{HAL Id: hal-02565340 \\ https://hal.science/hal-02565340}

Submitted on 6 May 2020

HAL is a multi-disciplinary open access archive for the deposit and dissemination of scientific research documents, whether they are published or not. The documents may come from teaching and research institutions in France or abroad, or from public or private research centers.
L'archive ouverte pluridisciplinaire HAL, est destinée au dépôt et à la diffusion de documents scientifiques de niveau recherche, publiés ou non, émanant des établissements d'enseignement et de recherche français ou étrangers, des laboratoires publics ou privés. 


\title{
Delay-induced instability in phase-locked dual-polarization distributed-feedback fiber lasers
}

\author{
M. Guionie, ${ }^{1}$ M. Romanelli, ${ }^{1}$ A. Thorette, ${ }^{1}$ A. Carré,${ }^{1}$ E. Pinsard, ${ }^{2}$ \\ L. Lablonde ${ }^{2}$ B. Cadier,${ }^{2}$ M. Alouini, ${ }^{1}$ M. Vallet, ${ }^{1}$ and M. Brunel ${ }^{1}$ \\ ${ }^{1}$ Univ Rennes, CNRS, Institut FOTON UMR 6082, F-35000 Rennes, France and \\ ${ }^{2}$ iXblue Photonics, rue Paul Sabatier, F-22300 Lannion, France
}

(Dated: May 6, 2020)

\begin{abstract}
We study experimentally and theoretically a dual-polarization fiber laser submitted to timedelayed frequency-shifted optical feedback. In addition to the usual frequency-locking regime that is expected when the frequency shift is close to the polarization beat frequency, we observe at high pumping rates a dynamical pulsing regime inside the locking range. This regime is experimentally evidenced in a full-fibered experiment based on an erbium-doped distributed-feedback fiber laser in which polarization beat frequency is about $1 \mathrm{GHz}$. A rate-equation model including the frequencyshifted feedback term reproduces well the experimental bifurcation maps, provided that both the time delay and a phase-amplitude coupling parameter ( $\alpha$ factor) are taken into account. The impact on microwave-photonics applications is discussed.
\end{abstract}

\section{INTRODUCTION}

Distributed-feedback (DFB) fiber lasers are appealing sources for various applications, notably because they offer a combination of low linewidth and high power [1]. When operating in a regime of simultaneous emission of two orthogonal eigenstates, such short-cavity lasers can be considered as microwave-optical sources, since the typical beat frequency between the two polarization modes is in the $\mathrm{GHz}$ range [2-9]. In this context, the stabilization of the beat frequency, in order to reduce the phase noise in microwave photonic links, is a question that led recently to develop an optical phase-locked loop scheme [9], and to the use of frequency-shifted optical feedback [10]. However a full dynamical description of fiber lasers with FSF has not been explored yet.

Frequency-shifted feedback (FSF) was originally introduced to stabilize dual-frequency bulk solid-state lasers [11, 12]. It fostered further developments in our group, leading to the discovery of original boundedphase [13, 14], excitablelike [15], or intensity-chaotic frequency-locked [16] regimes. Other studies involved integrated pairs of semiconductor lasers [17, 18], but the case of fiber lasers was left open. Contrary to bulk solidstate lasers, as we will show in the following, the effect of the time delay on the feedback regimes in DFB fiber lasers turns out to be important, as in many other fields [19]. Besides, the phase-amplitude coupling parameter ( $\alpha$ factor) remains a debated question for fiber lasers [20-23]. Since this parameter is well known to induce a wealth of dynamics in semiconductor lasers [24], it may also play a role in the fiber laser feedback scheme. Also, DFB fiber lasers were shown to exhibit specific dynamics with respect to semiconductor lasers in direct injection experiments [25, 26]. Consequently, the study of DFB fiber lasers subjected to optical feedback needs to be performed for both applied and fundamental laser dynamics issues.

Here we propose an all-fibered experimental setup based on a dual-frequency erbium-doped DFB fiber laser. A feedback loop containing an intensity modulator permits to reinject one frequency-shifted polarization mode into the other. In Sec. II, we show experimentally that short-cavity fiber lasers exhibit either a simple locking regime, or an original dynamical regime within the locking range, depending on the laser pumping rate. Phase noise is measured in the locking region. In Sec. III, we focus on a set of delayed-differential equations in order to model the system with the best accuracy, paying attention to the bifurcation maps obtained in the feedback rate-detuning plane and to the role of delay and $\alpha$ factor. Conclusions, discussion of applications, and perspectives are given in Sec. IV.

\section{EXPERIMENTAL RESULTS}

\section{A. Method}

The experimental set-up designed for the FSF stabilization of a DFB fiber laser is described in Fig. 1. The fiber laser is a 33 -mm-long $\mathrm{Er}^{3+}$-doped fiber into which a phase-shifted Bragg grating was photoinduced. The intensity transmission coefficients of the resulting mirrors linked to both grating sections separated by the phase step are estimated to be $-34 \mathrm{~dB}$ and $-57 \mathrm{~dB}$, respectively, and the laser effective length is about $2.6 \mathrm{~mm}$. The laser is pumped at $976 \mathrm{~nm}$ through a pump-signal combiner (WDM) and emits at $1547 \mathrm{~nm}$ two orthogonal polarization modes $E_{x}$ and $E_{y}$ with eigenfrequencies $\nu_{x}$ and $\nu_{y}$ respectively (we choose $\nu_{x}>\nu_{y}$ ). The threshold pump power is about $10 \mathrm{~mW}$, and the laser emits a total continuous-wave output power of about $100 \mu \mathrm{W}$ when backward-pumped with $100 \mathrm{~mW}$. Due to the birefringence induced by UV photo inscription of the Bragg grating [27], the polarization beat is at around $f_{b}=\nu_{x}-\nu_{y}=1 \mathrm{GHz}$. This beat frequency is slightly tunable using either the pump power, with a slope of 
$-20 \mathrm{kHz} / \mathrm{mW}$, or the DFB temperature, with a slope of about $10 \mathrm{kHz} / \mathrm{K}$. In free-running operation, the radiofrequency beat note has a linewidth of $3 \mathrm{kHz}$, and drifts within a few $\mathrm{MHz}$ span over a period of one day [9]. Such dual-frequency fiber laser (DFFL) free-running features are very attractive for heterodyning applications, compared to the use of separate lasers. However, it does not meet the standards for high-purity microwave-photonic links, hence motivating the search for efficient stabilization loops.
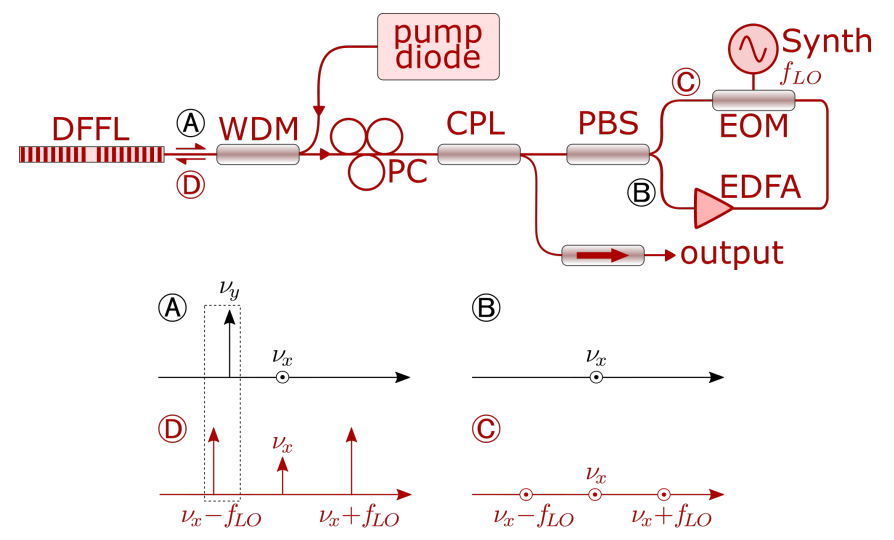

FIG. 1. Full-fibered experimental scheme of a dualfrequency fiber laser (DFFL) submitted to polarizationrotated frequency-shifted feedback. WDM, pump signal combiner; PC, polarization controller; CPL, 10-dB coupler; PBS, polarization beam splitter; EOM, electro-optic modulator driven by a synthesizer at frequency $f_{L O}$. The EDFA permits one to control the feedback power. Optical spectra at different points are sketched below: when $\nu_{x}$ is selected by the PBS, the $\nu_{x}-f_{L O}$ component of the reinjected field is resonant with $\nu_{y}$. See text for details.

The FSF loop is based on a polarization-maintaining (PM) polarization beam splitter whose outputs are closed on one another after passing through the frequency shifter, here realized with a PM electro-optic modulator (EOM). It is a standard Mach-Zehnder-type amplitude modulator which provides a high bandwidth $(10 \mathrm{GHz})$. The polarization controller $\mathrm{PC}$ between the laser and the FSF loop permits one to select one of the polarization states, say $E_{x}\left(\nu_{x}\right)$, with an extinction ratio reaching $40 \mathrm{~dB}$ (at point $\mathrm{B}$ in Fig. 1). The remaining circulating mode can be amplified through a PM erbiumdoped fiber amplifier (EDFA) with a maximum gain $G$ of $17 \mathrm{~dB}$, and then reaches the EOM. The EOM is driven by a synthesizer at frequency $f_{L O}$, acting as a local oscillator, and thus generates in the optical field two sidebands at frequencies $\nu_{x} \pm f_{L O}$. By biasing the EOM at minimum carrier transmission, the sideband power is maximized with respect to the carrier, with a typical 20$\mathrm{dB}$ ratio (at point $\mathrm{C}$ in Fig. 1). After the $\mathrm{PBS}$, we are then left with a feedback field whose polarization direction is parallel to $E_{y}$. Hence, for a small detuning $\Delta=f_{L O}-\left(\nu_{x}-\nu_{y}\right)=f_{L O}-f_{b}$, the reinjected field contains an optical sideband resonant with $\nu_{y}$. Intracavity coupling may then lead to frequency locking between $\nu_{y}$ and the $\nu_{x}-f_{L O}$ component of the reinjected field (compare $\mathrm{D}$ with $\mathrm{A}$ in Fig. 1). We then expect to find a stability transfer from the synthesizer $f_{L O}$ to the beat note $f_{b}$. When the EDFA is inserted, the overall feedback loop is about $8 \mathrm{~m}$, corresponding to a delay $\tau=40 \mathrm{~ns}$. Note that the clockwise rotation of the y polarization along the feedback loop is prevented by an optical isolator associated with the EDFA, thus eliminating any risks of mutual delayed feedback. Besides in order to monitor the beat note, a 90:10 optical coupler is inserted between the PC and the PBS. The $-10 \mathrm{~dB}$ output is then detected with a 10- $\mathrm{GHz}$ bandwidth photodiode after an optical isolator.

In addition to the detuning $\Delta$ parameter, two other system parameters are important in the following dynamical study: the injection rate and the laser pumping power. The injection rate is $\Gamma_{\text {exp }}=\sqrt{P_{i n j} / P_{x}}$, where $P_{x}$ is the intracavity power emitted in the $x$-polarized mode (roughly half the total power), and $P_{i n j}$ is the power reinjected inside the laser by the feedback field, that is, $y$ polarized and frequency shifted. Considering the estimated output coupler intensity transmission $T$ and the measured global transmission $T_{\text {loop }}$ of the feedback loop (including EOM conversion losses as well as all the insertion losses), the EDFA gain parameter permits one to raise $\Gamma_{\text {exp }}=T \sqrt{T_{\text {loop }} G}$ up to $3.8 \times 10^{-4}$. Note that, while the reinjected optical power is very low (at the $\mathrm{pW}$ level), it still corresponds to a strong feedback: the normalized reinjection rate as defined in Sec. III is found to be in the $0-30$ range. Besides, the pumping power is commonly characterized by the excitation rate $\eta$ which is the ratio of the pumping power to the threshold power.

\section{B. Stabilization results}

In a first step, we record the RF spectra when the local oscillator is swept. We choose to inject $E_{x}$ into the FSF loop, as in Fig. 1, with a moderate excitation rate $\eta=1.3$ and an EDFA gain of $12 \mathrm{~dB}$ yielding $\Gamma_{\exp }=2.2 \times 10^{-4}$. The free-running beat is $f_{b}=1.015 \mathrm{GHz}$. In this case, the spectrogram shown in Fig. 2(a) is recorded while sweeping the synthesizer frequency across $f_{b}$ over a 7-MHz span in 1000 steps. As a result, each of the 1000 lines in the figure corresponds to an electrical spectrum coded in false colors. The two (high-intensity) yellow lines correspond to the free-running beat $f_{b}$ (central line) and the swept synthesizer $f_{L O}$ (oblique line), respectively. As expected from such an injection experiment, when the local oscillator is close enough to the free beat note, the laser gets locked. Figures 2(b) and 2(c) show spectra corresponding to two lines of Fig. 2(a), in the unlocked and locked regimes, respectively. The locking range between the beat note and the synthesizer, emphasized by the dotted box in Figs. 2(a) and 2(d), is measured to be about $2 \mathrm{MHz}$.

We observe that the locking region increases with the reinjection rate, as is usual with injection schemes. Fig- 

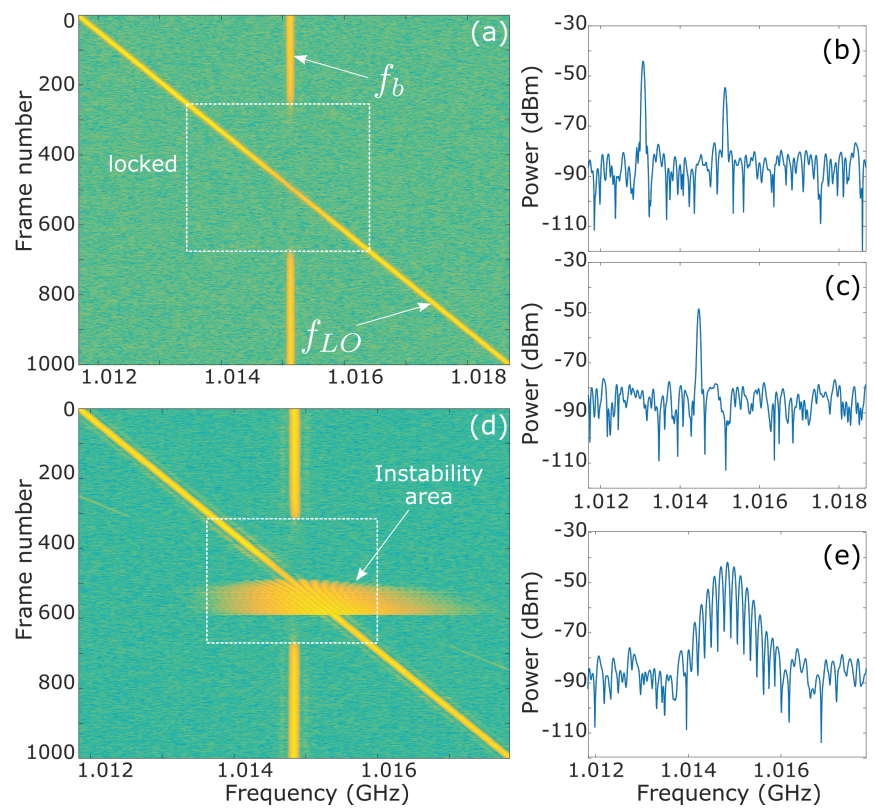

FIG. 2. RF spectrum analysis of the beat note. $\Gamma_{\exp }=$ $2.2 \times 10^{-4}(G=12 \mathrm{~dB})$. Resolution bandwidth (RBW) $50 \mathrm{kHz}$. (a)-(c) $\eta=1.3(\Gamma=20)$, Span $7 \mathrm{MHz}$. (a) Swept spectrogram showing unlocked and locked states. RF spectra (b) from the unlocked regime and (c) from the locked regime in (a). (d) and (e) $\eta=4.1(\Gamma=12)$. Span $6 \mathrm{MHz}$. (d) Swept spectrogram showing the instability area within the locked state. (e) RF spectrum extracted from the instability area in (d).

ure 3 shows a map of the experimental locking region in the $(\Delta, \Gamma)$ plane. $\Gamma$ is driven by the EDFA gain. At a maximum gain of $17 \mathrm{~dB}$, the locking range is $4-\mathrm{MHz}$ wide. It is noteworthy to mention that such a locking range is wide enough to compensate for usual laboratory temperature variations, and that the DFFL then stays locked for days. This map also shows that the locking region is symmetrical with respect to the detuning. Note also that, in a first realization of the loop made without the EDFA, stabilization also occurred at small detunings and the locking range was measured to be about $200 \mathrm{kHz}$.

\section{Self-pulsing dynamics}

In a second step, we notice that the DFFL offers another kind of response at higher pumping rates. Indeed, we observe an area of instabilities inside the locking range as soon as $\eta$ is above a value of roughly 3 . The corresponding spectrogram is then the one shown in Fig. 2(d), recorded at $\eta=4.1$, for example. The instability area appears for positive values of $\Delta$, and the spectrum then develops a comb of equidistant lines with a frequency spacing of the same order of magnitude as the relaxation oscillation frequency $\left(f_{R}=170 \mathrm{kHz}\right.$ in this case), as depicted in Fig. 2(e). The corresponding oscilloscope trace is reproduced in Fig. 4, showing a regular self-pulsing regime with a $200-\mathrm{kHz}$ repetition rate of $2-\mu \mathrm{s}-$ long pulses.

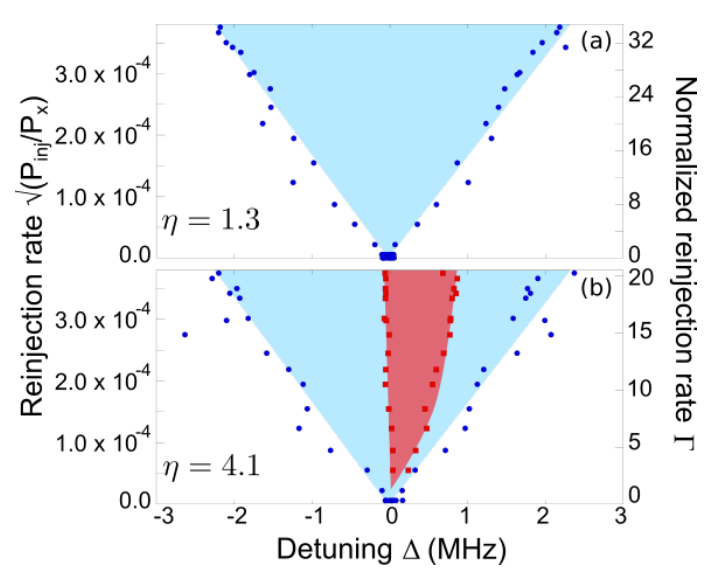

FIG. 3. Experimental stability maps in the $(\Delta, \Gamma)$ plane at different pumping rates. (a) $\eta=1.3$, (b) $\eta=4.1$. Dots are experimental points. Colored areas delimit the different stability regimes. White, unlocked; blue, locked; red, selfpulsing instability.

We observe that the pulsing period increases with the detuning. The lower panel of Fig. 3 shows both locking (in blue) and instability (in red) regions in the $(\Gamma, \Delta)$ plane for $\eta=4.1$. As in the previous low excitation rate case, the locking range increases with the injection rate up to about $4 \mathrm{MHz}$ at maximum. The normalized righthand side vertical scale changes with respect to the upper panel, since the relaxation oscillation frequency increases with the excitation rate.

Interestingly, we find that when the roles of $E_{x}$ and $E_{y}$ are interchanged, the response of the system looks different at first sight. Thanks to the polarization controller, we check the dynamical responses when $E_{y}$ is injected in the FSF loop instead of $E_{x}$. In this case, frequency locking occurs between $\nu_{x}$ and the $\nu_{y}+f_{L O}$ component of the reinjected field. We still find a locking region, with a comparable width, but the instability area appears for an opposite detuning. This is obvious by comparing the spectrograms of Figs. 5(a) and 5(b). This apparent sign reversal is explained by considering the optical frequencies: In the first situation [Figs. 5(a)], the instability is obtained when $\Delta>0$, that is $\nu_{x}-f_{L O}<\nu_{y}$; in the second situation [Figs. 5(b)] the instability is obtained when $\Delta<0$, that is $\nu_{y}+f_{L O}<\nu_{x}$. It means that, in both cases, the instability area is consistently found when the reinjected "master" optical frequency is lower than the "slave" optical frequency. Another feature of the instability area is that it appears whatever the sweeping direction but with a slight hysteresis: The width of this instability area is narrower when $\Delta$ decreases in the first situation $\left[\nu_{x}-f_{L O}\right.$ injects $\nu_{y}$; see Fig. $5($ a) $]$, or when $\Delta$ increases in the second situation $\left[\nu_{y}+f_{L O}\right.$ injects $\nu_{x}$; see Fig. 5(b)].

Finally, when the DFFL operates within the stability region, one can measure its phase noise. In Fig. 6, we plot the phase noise spectra of the beat note in two different parameter regions: in the phase-locked state 


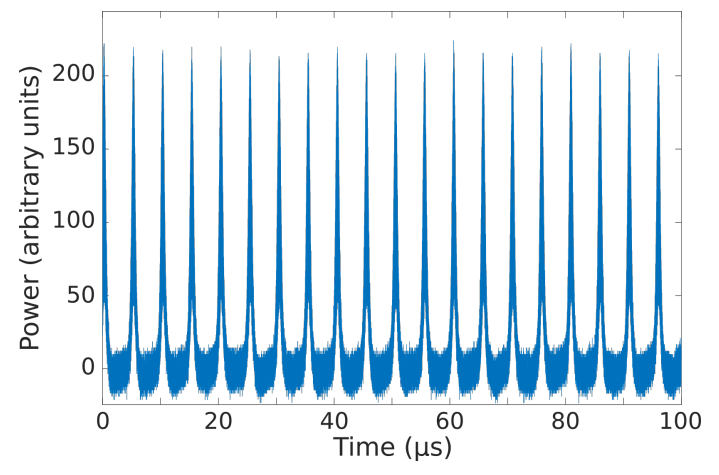

FIG. 4. Oscilloscope trace of the self-pulsing regime inside the instability area. $\eta=11.2$ and $\Gamma=5$.
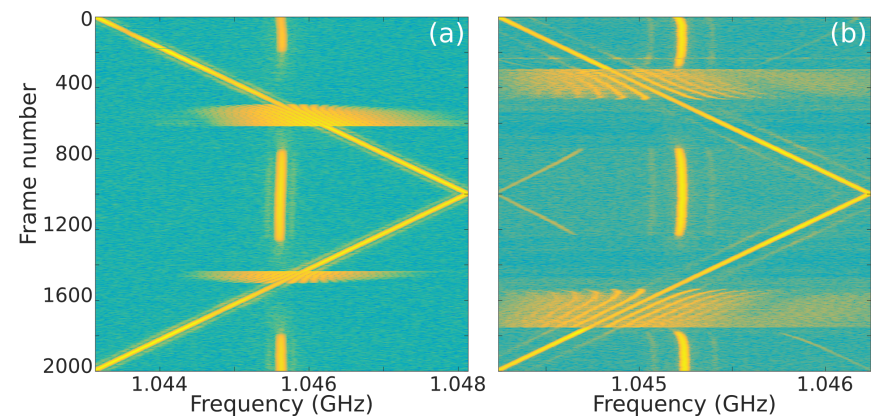

FIG. 5. RF spectrograms recorded $\eta=4.1$ and $G=13.6 \mathrm{~dB}$. (a) $E_{x}$ reinjected with $\Gamma_{e x p}=2.6 \times 10^{-4}(\Gamma=13.9)$. Span $5 \mathrm{MHz}$. Instability areas are observed when $\Delta>0$. (b) $E_{y}$ reinjected with $\Gamma_{\text {exp }}=1.9 \times 10^{-4}(\Gamma=10.9)$. Span $2 \mathrm{MHz}$. Instability areas observed when $\Delta<0$.

(green curve) and at the edge of the instability region (blue curve). One can see that the two curves are close to each other at low offset frequencies; this could be an indication of a bounded-phase state at the edge of the instability area [16]. In the instability area, the phase noise spectrum is affected by relaxation oscillations and harmonics, but it is strongly reduced to $-80 \mathrm{dBc} / \mathrm{Hz}$ at $1 \mathrm{kHz}$ offset. These measurements are compared with a previous plot of the free-running beat note phase noise [9]: The noise is reduced by $40 \mathrm{~dB}$ at $1-\mathrm{kHz}$ offset even in the instability region.

To the best of our knowledge, this dynamical instability had not been observed in other laser structures, and it is surely related to the specific time scales of this system. We develop a rate-equation model in the next section that intends to support these original experimental observations.

\section{THEORETICAL MODEL}

\section{A. Rate equations with delayed feedback}

In order to reproduce the experimental results, we introduce the following model equations:

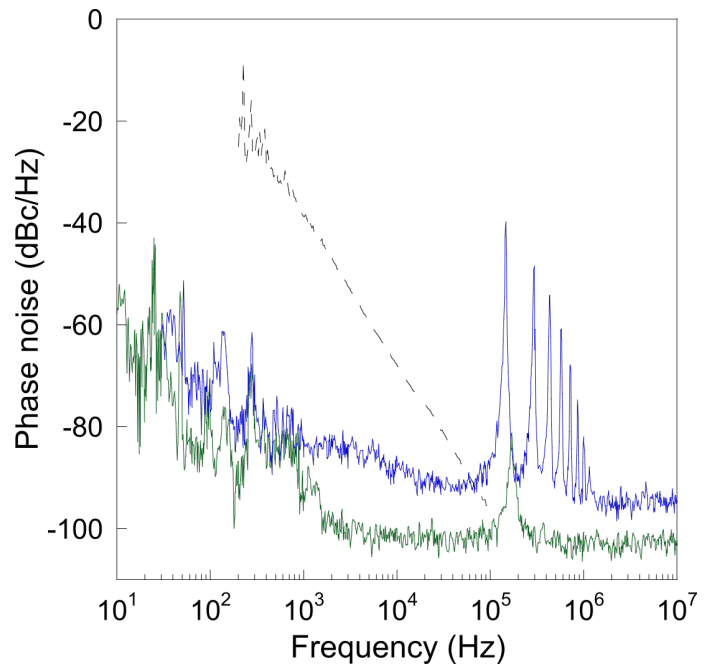

FIG. 6. Phase noise of the beat note in the locked state (green curve), and at the edge of the self-pulsing state (blue curve). Phase noise of the free-running beat, obtained from the phase-locked loop in [9] is recalled for comparison (dashed black curve).

$$
\begin{aligned}
\frac{d e_{x}}{d s} & =(1+i \alpha) \frac{\left(m_{x}+\beta m_{y}\right)}{1+\beta} \frac{e_{x}}{2}, \\
\frac{d e_{y}}{d s} & =(1+i \alpha) \frac{\left(m_{y}+\beta m_{x}\right)}{1+\beta} \frac{e_{y}}{2} \\
& +i \Delta e_{y}+\Gamma e_{x}(t-\tau), \\
\frac{d m_{x, y}}{d s} & =1-\left(\left|e_{x, y}\right|^{2}+\beta\left|e_{y, x}\right|^{2}\right) \\
& -\epsilon m_{x, y}\left[1+(\eta-1)\left(\left|e_{x, y}\right|^{2}+\beta\left|e_{y, x}\right|^{2}\right)\right] .
\end{aligned}
$$

The model describes the amplitudes $e_{x, y}$ of two laser fields coupled by optical injection, and the relative population inversions $m_{x, y}$. The scaled time $s$ is related to the physical time $t$ by $s=2 \pi f_{R} t . \eta$ is the pump parameter, and $\epsilon=\sqrt{\frac{\tau_{c a v}}{(\eta-1) \tau_{i n v}}}$, where $\tau_{c a v}$ and $\tau_{i n v}$ are the cavity and inversion lifetimes, respectively. $\beta$ accounts for cross-saturation in the active medium. The $e_{x}$ field is injected into the $e_{y}$ field after having been delayed and frequency shifted. The injection process is accounted for by two parameters: the detuning $\Delta$ and the injection strength $\Gamma$. The assumptions and approximations leading to this model are described in more detail in $[14,15]$ and references therein. With respect to the equations written in $[14,15]$, two important parameters complete the model: The equations contain the phase-amplitude coupling $\alpha$ and the delay $\tau$ associated with the propagation time in the fibered feedback loop.

The model parameters are set as follows. $\tau_{i n v}$ is estimated from the transient response of the laser to a step in the pumping process, similarly to what is done in [28]. Combining the estimated value $\tau_{\text {inv }} \simeq 75 \mu \mathrm{s}$ with the measure of $f_{R}$, around $160 \mathrm{kHz}$ for a pump rate $\eta=4$, 

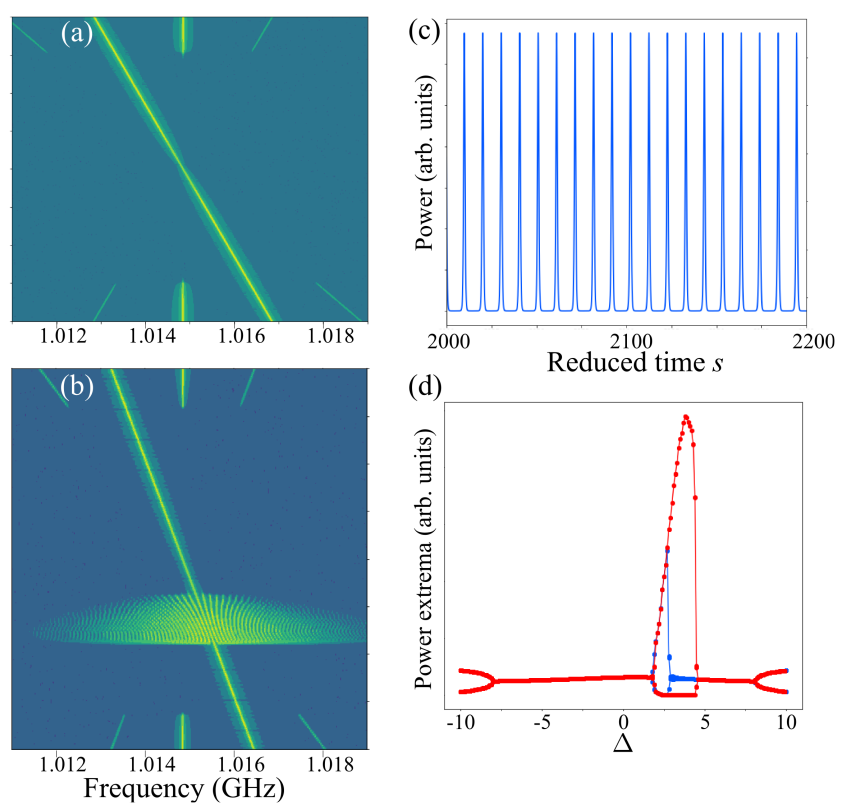

FIG. 7. RF spectrogram computed from Eqs. (1)-(3) with (a) $\eta=1.3, \Gamma=15, \tau=0.029, \epsilon=0.085$; and (b) $\eta=4.1, \Gamma=$ $8, \tau=0.047, \epsilon=0.0133$. (c) Time series inside the instability area with $\eta=4.1$ and $\Delta=3$. (d) Bifurcation diagram as $\Delta$ is swept from left to right (red), then from right to left (blue), showing hysteresis as in the experiment (Fig. 5). $\alpha=1$ and $\beta=0.65$ in all simulations.

one can deduce $\tau_{c a v} \simeq 40$ ns. We note that from $\tau_{c a v}$ one can evaluate the global losses over one roundtrip to be $6 \times 10^{-4}(-32 \mathrm{~dB})$. This is in good agreement with the independently estimated mirror transmission of $-34 \mathrm{~dB}$. The reinjection strength $\Gamma$ can be calculated via its expression $\Gamma=\frac{\Gamma_{e x p}}{\tau_{R T} 2 \pi f_{R}}$, where $\tau_{R T}$ is the roundtrip time inside the laser cavity. For a typical value of $G=11 \mathrm{~dB}$ one gets $\Gamma \simeq 11$. Now, $\Gamma$ also gives an estimate of the half width of the locking range normalized to $f_{R}$. This leads us to predict a locking range of around $3 \mathrm{MHz}$, in good agreement with experiments. An important parameter ruling the dynamics of the system is the cross-saturation parameter $\beta$. We measure it experimentally by using the ratio of the antiphase and relaxation oscillation frequencies $[29,30]$ and find $\beta=0.65$, in agreement with former measures in bulk erbium lasers [31].

\section{B. Simulation results}

Figures $7(\mathrm{a})$ and $7(\mathrm{~b})$ present the power spectrum of the beat-note intensity $I_{x y}$ as a function of the detuning $\Delta$. Given that Eqs. (1)-(3) are written for slowly varying amplitudes, the interference between the $x-$ and $y-$ polarized fields must be written as [14]

$$
I_{x y}=\left|e_{x} e^{i 2 \pi \nu_{x} s}+e_{y} e^{i 2 \pi\left(\nu_{x}-f_{L O}\right) s}\right|^{2}
$$
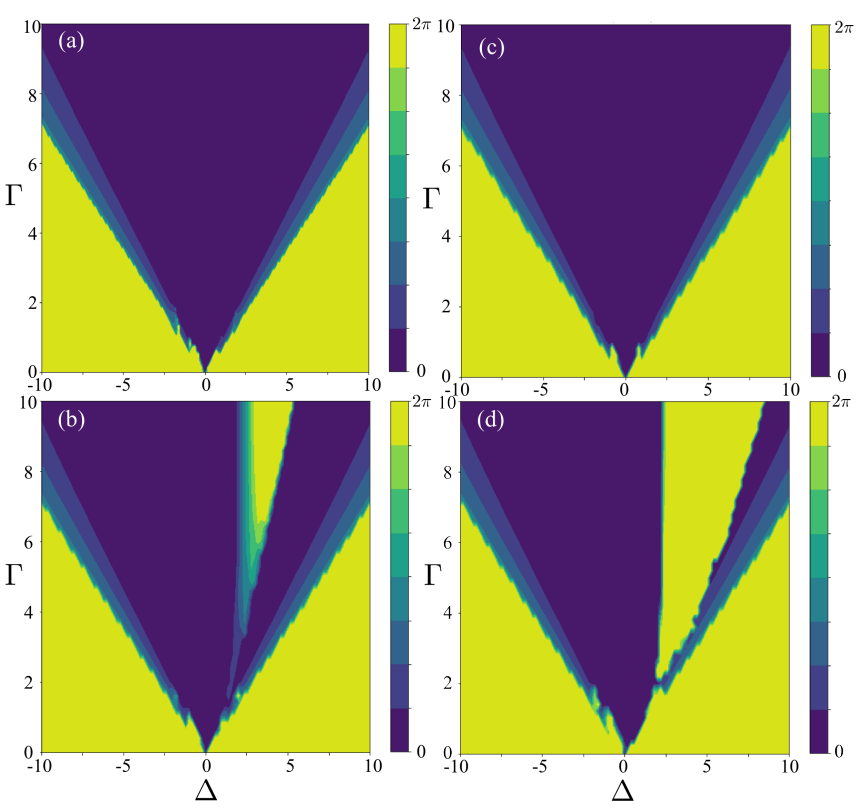

FIG. 8. Simulated stability maps in the $(\Delta, \Gamma)$ plane at (a) $\eta$ $=1.3$ and $\alpha=1$; (b) $\eta=4.1$ and $\alpha=1$; (c) $\eta=4.1$ and $\alpha=$ 0 ; and (d) $\eta=4.1$ and $\alpha=2$. Other parameters as in Fig. 7 .

$$
=\left|e_{x}+e_{y} e^{-i 2 \pi\left[f_{b}+\Delta\right] s}\right|^{2}
$$

where all frequencies are normalized to $f_{R}$. The numerical value of the free-running beat note frequency $f_{b}$ is immaterial for the present discussion, and has been shifted to about $1.015 \mathrm{GHz}$ in Fig. 7 to match with the experimental value. We can see from Fig. 7 that the model is able to reproduce the experimental findings. When $|\Delta|$ becomes smaller than $\Gamma$, the beat-note frequency locks on $f_{L O}$. Furthermore, at higher pumping rates, we recover an instability area inside the locking range, for positive detunings only, as in the experiment. Inside the instability area, observed periodic self-pulsing regime is predicted as well, as shown in Fig. 7 (c). This self-pulsing regime appears for moderate, positive detunings only, and presents some hysteresis [Fig. 7 (d)]. As the detuning is further increased, phase-locking with the local oscillator is recovered.

In order to have a more global view, we have computed maps of the system's behavior in the $\{\Delta, \Gamma\}$ plane (Fig. 8). To obtain these maps, a time series is computed for each value of $\Delta$ and $\Gamma$. Then, after eliminating the transient dynamics, we calculate the difference between the extrema of $\delta \phi(t)=\phi_{y}(t)-\phi_{x}(t)$, where $\phi_{y}(t)$ and $\phi_{x}(t)$ are the arguments of $e_{y}$ and $e_{x}$, respectively. If $\max (\delta \phi)-\min (\delta \phi)=0$ then the system is phase locked, corresponding to the dark regions in the maps. Conversely if $\max (\delta \phi)-\min (\delta \phi)=2 \pi$ then the system is unlocked, as in the yellow regions. Intermediate boundedphase zones where $\max (\delta \phi)-\min (\delta \phi)<2 \pi$ also appear. Figures $8(\mathrm{a})$ and $8(\mathrm{~b})$ are computed with the same phase-amplitude coupling $\alpha=1$. From Fig. 8(a), which is computed with $\eta=1.3$, we can see that the instability 


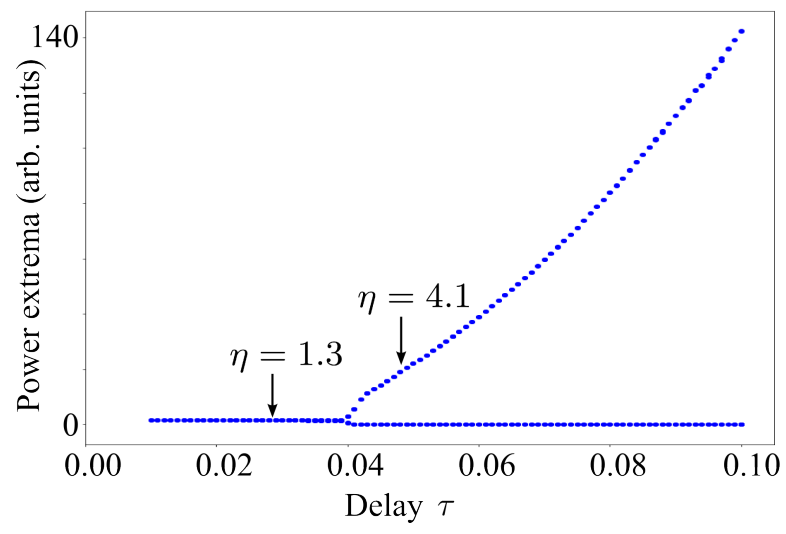

FIG. 9. Bifurcation diagram of the beat-note envelope vs the delay $\tau . \eta=4.1, \Gamma=8, \Delta=3$.

area disappears at low pump rates. Figure $8(\mathrm{~b})$ is computed with $\eta=4.1$ and reproduces the appearance of the instability area. We check the influence of the $\alpha$ parameter on these results. First, Fig. 8(c) computed with $\alpha$ $=0$ fails to reproduce the experimental results, since the instability area disappears. Second, Fig. 8(d) computed with $\alpha=2$ shows a wide instability zone as in the experiment but the bounded-phase zone almost disappears. In Fig. 8(b) we notice that, close to the left border of the instability area, we have a region of bounded phase in agreement with the measured phase noise in Fig. 6, a behavior already reported in other lasers [32]. Concerning the apparent sign reversal observed experimentally in Figs. 5(a) and 5(b), we notice that, in Eqs. (1)-(3), whether $\nu_{x}>\nu_{y}$ or not, the form of the equations is the same. However, if $\nu_{x}>\nu_{y}$ then $\nu_{i n j}=\nu_{x}-f_{L O}$, and we obtain the RF detuning $\Delta=f_{L O}-f_{b}$. The instability occurs for $\Delta>0$, i.e., for $f_{L O}>f_{b}$. If, on the contrary, one has $\nu_{x}<\nu_{y}$, then $\nu_{i n j}=\nu_{x}+f_{L O}$, and $\Delta$ becomes $\Delta=f_{b}-f_{L O}$. So, in this latter case the instability appears for $f_{L O}<f_{b}$.

\section{Discussion}

What do we learn from simulations, that was not known from the experiment? A first point is that the phase-amplitude $\alpha$ factor, which is sometimes overlooked in solid-state lasers, cannot be ignored in order to reproduce the experimental findings, in particular the asymmetric behavior with respect to $\Delta$. We notice that a moderate value $\alpha=1$ yields a set of simulations consistent with the experimental data. Another unexpected insight is that the seemingly short delay $(\tau \simeq 0.05$ in units of the laser intrinsic time scale $1 / \omega_{R}$ ) plays a crucial role in the appearance of the instability. This is obvious from the bifurcation diagram in Fig. 9, where we see that the instability begins at a finite, nonzero value of the delay. This also sheds light on the dependence on the pump rate $\eta$. Indeed, a lower value of $\eta$ implies a slower laser intrinsic dynamics, so that effectively, at low pump rates, the feedback loop appears shorter $(\tau=0.029$ for $\eta=1.3$, while $\tau=0.047$ for $\eta=4.1$ ) and the instability disappears. Finally the crucial role of the delay also reveals that the coupling due to cross-saturation in the active medium is essential for observing this instability. Indeed, if $\beta=0$ the system boils down to a master-slave configuration where the $x$ mode is unaffected by the dynamics so that $e_{x}$ is a constant: Obviously in this case the notion of delay loses its meaning.

\section{CONCLUSION}

We have studied experimentally and theoretically a dual-polarization fiber laser in the presence of frequencyshifted feedback. In addition to the expected phaselocking effect typical of coupled oscillators, we have observed an original dynamical regime of self-pulsed operation inside the phase-locking region. A set of normalized coupled rate equations has been introduced with all the parameters experimentally measured except the $\alpha$ factor. It permits one to find all the typical regimes of this laser's dynamics: the phase locking region and the instability area.

As regards microwave photonics applications, our feedback scheme provides a very efficient stabilization mechanism of the beat note. The locking range can reach $4 \mathrm{MHz}$ at maximum gain in our setup; it could be further enhanced with higher optical gain in the loop. The locking range is wide enough to compensate for environmental drifts, leading to robust beat-note stabilization for days, with a phase noise as low as $-90 \mathrm{dBc} / \mathrm{Hz}$ at $1-\mathrm{kHz}$ offset frequency. Here experiments were conducted with a beat note around $1 \mathrm{GHz}$, but the loop would work at higher frequencies if necessary, provided a wide-bandwidth modulator such as the EOM is used. Finally the setup is all-fibered and could be integrated in a compact system.

Our work also shows that the phase-locked state can be destabilized into a regular self-pulsing state, even with a modest delay. We have found that this instability area is characterized by the following features: (i) It appears when the reinjected optical frequency is lower than the other laser frequency only (denoting a nonzero value of the $\alpha$ factor), (ii) its width increases with the reinjection rate, (iii) it appears whatever the sweeping direction but with a slight hysteresis. This impacts of course the design of such loops for applications, where shorter loops would be preferable to avoid spurious self-pulsed states.

Finally our rate-equation model provides simulations consistent with all the experimental results, showing in particular the necessity to include the $\alpha$ factor and the feedback loop time delay. Extension to longer delays can be conveniently realized in our all-fibered laser platform, and easily implemented in the simulations for comparison. This model could also provide a basis for further study of other short-cavity dual-frequency lasers, such as DBR fiber lasers [8] or VECSELs [33]. 


\section{ACKNOWLEDGMENTS}

This work was partially funded by DGA (Grants No. ANR-16-ASTR-0016 and No. 2017-60-0010), and Ré- gion Bretagne, FEDER, and Rennes Metropole (CPER SOPHIE-Photonique).
[1] S. Fu, W. Shi, Y. Feng, L. Zhang, Z. Yang, S. Xu, X. Zhu, R. A. Norwood, and N. Peyghambarian, Review of recent progress on single-frequency fiber lasers, J. Opt. Soc. Am. B 34, A49 (2017).

[2] W. H. Loh and R. I. Laming, $1.55 \mu \mathrm{m}$ phase-shifted distributed-feedback fiber laser, Electron. Lett. 31, 1440 (1995).

[3] J. S. Leng, Y. C. Lai, W. Zhang, and J. A. R. Williams, A new method for microwave generation and data transmission using DFB laser based on fiber Bragg gratings, IEEE Photon. Technol. Lett. 18, 1729 (2006).

[4] B.-O. Guan, Y. Zhang, L.-W. Zhang, and H.Y. Tam, Electrically tunable microwave generation using compact dual-polarization fiber laser, IEEE Photon. Technol. Lett. 21, 727 (2009).

[5] Y.-N. Tan, L. Jin, L. Cheng, Z. Quan, M. Li, and B.-O. Guan, Multi-octave tunable RF signal generation based on a dual-polarization fiber grating laser, Opt. Express 20, 6961 (2012).

[6] J. Maxin, S. Molin, G. Pillet, L. Morvan, A. Mugnier, D. Pureur and D. Dolfi, Dual-frequency distributed feedback fibre laser for microwave signals generation, Electron. Lett. 47, 816 (2011).

[7] Q. Yuan, Y. Liang, L. Jin, L. Cheng, and B.-O. Guan, Implementation of a widely tunable microwave signal generator based on dual-polarization fiber grating laser, Appl. Opt. 54, 895 (2015).

[8] Y. Liang, L. Jin, L. Cheng, and B.-O. Guan, Stabilization of microwave signal generated by a dual-polarization DBR fiber laser via optical feedback, Opt. Express 22, 29356 (2014).

[9] M. Guionie, L. Frein, F. Bondu, A. Carré, G. Loas, E. Pinsard, L. Lablonde, B. Cadier, M. Alouini, M. Romanelli, M. Vallet, and M. Brunel, Beat note stabilization in dual-polarization DFB fiber lasers by an optical phase-locked loop, Opt. Express 26, 3483 (2018).

[10] M. Guionie, A. Thorette, M. Romanelli, A. Carré, G. Loas, E. Pinsard, L. Lablonde, B. Cadier, M. Alouini, M. Vallet, and M. Brunel, "Microwave-optical fiber lasers stabilized by frequency-shifted feedback," in IEEE International Topical Meeting on Microwave Photonics (IEEE, Piscataway, 2019).

[11] L. Kervevan, H. Gilles, S. Girard, and M. Laroche, Beatnote jitter suppression in a dual-frequency laser using optical feedback, Opt. Lett. 32, 1099 (2007).

[12] J. Thévenin, M. Vallet, M. Brunel, H. Gilles, and S. Girard, Beat-note locking in dual-polarization lasers submitted to frequency-shifted optical feedback, J. Opt. Soc. Am. B 28, 1104 (2011).

[13] J. Thévenin, M. Romanelli, M. Vallet, M. Brunel, and T. Erneux, Resonance Assisted Synchronization of Coupled Oscillators: Frequency Locking without Phase Locking, Phys. Rev. Lett. 107, 104101 (2011).

[14] J. Thévenin, M. Romanelli, M. Vallet, M. Brunel, and T. Erneux, Phase and intensity dynamics of a two-frequency laser submitted to resonant frequency-shifted feedback, Phys. Rev. A 86, 033815 (2012).

[15] M. Romanelli, A. Thorette, M. Brunel, T. Erneux, and M. Vallet, Excitable-like chaotic pulses in the boundedphase regime of an opto-rf oscillator, Phys. Rev. A 94, 043820, (2016).

[16] A. Thorette, M. Romanelli, M. Brunel, and M. Vallet, Frequency-locked chaotic opto-RF oscillator, Opt. Lett. 41, 2839 (2016).

[17] L. Wang, M. Romanelli, F. van Dijk, and M. Vallet, Photonic microwave oscillator based on monolithic DFB lasers with frequency- shifted feedback, Electron. Lett. 50, 451 (2014).

[18] M. Vallet, M. Romanelli, G. Loas, F. Van Dijk, and M. Alouini, Self-stabilized optoelectronic oscillator using frequency-shifted feedback and a delay line, IEEE Photon. Technol. Lett. 28, 1088 (2016).

[19] T. Erneux, J. Javaloyes, M. Wolfrum, and S. Yanchuk, Introduction to focus issue: Time-delay dynamics, Chaos 27, 114201 (2017).

[20] E. Rønnekleiv, Frequency and intensity noise of single frequency fiber Bragg grating lasers, Opt. Fiber Technol. 7, 206 (2001).

[21] S. Foster, Complex susceptibility of saturated erbiumdoped fiber lasers and amplifiers, IEEE Photon. Technol. Lett. 19, 895 (2007).

[22] S. Foster, G. A. Cranch, and A. Tikhomirov, Experimental evidence for the thermal origin of 1 /f frequency noise in erbium-doped fiber lasers, Phys. Rev. A 79, 053802 (2009).

[23] G. A. Cranch, G. A. Miller, J. Harrison, and S. Foster, Characterization of the DFB fiber laser resonator strength and $\alpha$-parameter by response to external feedback, in CLEO San Jose 9-14 June 2013 (IEEE, Piscataway, 2014).

[24] J. Ohtsubo, Semiconductor Lasers. Stability, Instability and Chaos (Springer, Berlin, 2017).

[25] S. Blin, Réalisation de lasers à fibre à contre-réaction répartie pour l'étude de l'injection optique: comparaison à l'injection avec des lasers à semi-conducteurs (Université de Rennes 1, Rennes, 2003).

[26] S. Blin, P. Besnard, O. Vaudel, and S. La Rochelle, Optical injection in semiconductor or fiber lasers: A comparison, the influence of coherence, Proc. SPIE 5452, 534 (2004).

[27] T. Erdogan and V. Mizrahi, Characterization of UVinduced birefringence in photosensitive Ge-doped silica optical fiber, J. Opt. Soc. Am. B 11, 2100 (1994).

[28] E. Lacot, F. Stoeckel, and M. Chenevier, Dynamics of an erbium-doped fiber laser, Phys. Rev. A 49, 3997 (1994).

[29] A. J. Poustie, Polarization cross saturation in an $\mathrm{Er}^{3+}$. doped fiber ring laser, Opt. Lett. 20, 1868 (1995).

[30] E. Lacot and F. Stoeckel, Nonlinear mode coupling in a microchip laser, J. Opt. Soc. Am. B 13, 2034 (1996). 
[31] M. Brunel, A. Amon, and M. Vallet, Dual-polarization microchip laser at $1.53 \mu \mathrm{m}$, Opt. Lett. 30, 2418 (2005).

[32] M. Romanelli, L. Wang, M. Brunel, and M. Vallet, Measuring the universal synchronization properties of driven oscillators across a Hopf instability, Opt. Express 22, 7364 (2014).
[33] G. Baili, L. Morvan, M. Alouini, D. Dolfi, F. Bretenaker, I. Sagnes, and A. Garnache, Experimental demonstration of a tunable dual-frequency semiconductor laser free of relaxation oscillations, Opt. Lett. 34, 3421 (2009). 